\title{
Proyectos Ambientales Escolares desde la Red verde de trabajo a través de las
} TIC

\section{School Environmental Projects from the Green Network of work through ICT}

\author{
Geidy Antonia Parejo-Blanco \\ geidyparejo@gmail.com \\ Universidad Popular del Cesar, Valledupar \\ Colombia \\ https://orcid.org/0000-0003-1460-5695 \\ Amarilis Esther Martínez-Viloria \\ amesmavi2014@gmail.com \\ Universidad Popular del Cesar, Valledupar \\ Colombia \\ https://orcid.org/0000-0001-5617-5887 \\ María-del-Carmen Jiménez-Barriosnuevo \\ mariacjimenez@unicesar.edu.co \\ Universidad Popular del Cesar, Valledupar \\ Colombia \\ https://orcid.org/0000-0002-2491-3731
}

Recibido: 03 de mayo de 2021

Aprobado: 04 de agosto de 2021 


\title{
RESUMEN
}

La investigación tuvo el propósito de crear una "Red Verde" de trabajo participativo mediado por las TIC, para la reconstrucción de los PRAE, en las instituciones educativas de la comuna 6 de Santa Marta, desde la IED Once de Noviembre como escenario piloto. Metodológicamente, se asumió un enfoque participativo, guiado por la Investigación Acción Transformadora y siguiendo su diseño de las fases: diagnóstico social participativo, planificación, ejecución y evaluación mediante el mecanismo de acciónreflexión-acción. La recolección y análisis de los datos se dio desde un enfoque mixto, utilizándose técnicas e instrumentos de corte cuantitativo y cualitativo. La "Red Verde" de trabajo participativo mediada por las TIC y la ruta para la reconstrucción de los PRAE, se constituye en una estrategia de EA pedagógicamente pertinente, pues, fomento el conocimiento y la gestión, de los problemas ambientales desde la realidad contextual, producto de las dinámicas naturales y socioculturales, de manera colaborativa.

Descriptores: Educación ambiental; tecnología; desarrollo participativo. (Palabras tomadas del Tesauro UNESCO).

\begin{abstract}
The research had the purpose of creating a "Green Network" of participatory work mediated by ICTs, for the reconstruction of the PRAE, in the educational institutions of the commune 6 of Santa Marta, from the IED Once de Noviembre as a pilot scenario. Methodologically, a participatory approach was assumed, guided by the Transformative Action Research and following its design of the phases: participatory social diagnosis, planning, execution and evaluation through the action-reflection-action mechanism. Data collection and analysis was done from a mixed approach, using quantitative and qualitative techniques and instruments. The "Green Network" of participatory work mediated by ICT and the route for the reconstruction of the PRAE, constitutes a pedagogically relevant EE strategy, therefore, it fosters knowledge and management of environmental problems from the contextual reality, product of natural and sociocultural dynamics, in a collaborative way.
\end{abstract}

Descriptors: Environmental education; technology; participatory development. (Words taken from the UNESCO Thesaurus). 


\section{CIENCIAMATRIA}

Revista Interdisciplinaria de Humanidades, Educación, Ciencia y Tecnología

Año VII. Vol. VII. N¹. Edición Especial. 2021

Hecho el depósito de ley: pp201602FA4721

ISSN-L: 2542-3029; ISSN: 2610-802X

Universidad Nacional Experimental Francisco de Miranda (UNEFM). Santa Ana de Coro. Venezuela

Geidy Parejo-Blanco; Amarilis Martínez-Viloria; María-del-Carmen Jiménez-Barriosnuevo

\section{INTRODUCCIÓN}

El crecimiento poblacional y el desarrollo socioeconómico trae consigo riesgos y consecuencias para todas las especies de la tierra. Es por ello que la Educación Ambiental (EA) es considerada una herramienta de cultura ambiental (Burgos, 2017); por consiguiente, y siendo uno de los medios más efectivos para concientizar a las personas sobre los impactos de su comportamiento en el medio ambiente, resulta fundamental formar la cultura ambiental, mediante el fortalecimiento de actitudes reflexivas y críticas desde la niñez y la juventud (Bustamante, Cruz \& Vergara, 2017).

Considerando lo expuesto, es de saber que Colombia acoge la dimensión ambiental mediante la formalización de la EA como materia de educación formal de todas las instituciones del país (Chequea \& Collazos, 2014); pero, premeditada desde las concepciones culturales y familiares, para que haya un sentido de pertenencia del entorno, por parte de las comunidades (Flórez, 2015). De allí que emergen los Proyectos Ambientales Escolares (PRAE), los cuales son estrategias que estructuran y entretejen la EA en el currículo institucional, con el fin de generar espacios de crítica y reflexión que promuevan habilidades en investigación, en torno a las problemáticas y las fortalezas medioambientales (Huérfano, 2018). Cabe resaltar que el MinEducación (2005), define los PRAE, como proyectos del ámbito pedagógico que buscan interpretar los problemas ambientales, a nivel local, sin desconocimiento de las situación regional y nacional, a través de espacios de participación que permitan implementar soluciones relacionadas con las dinámicas naturales y socioculturales.

Por tanto, para la construcción y ejecución del PRAE, enmarcado en lo que es el Proyecto Ambiental Colectivo como estrategia en el marco de la Política Ambiental del Plan de Desarrollo "Cambio para construir la paz" (MinAmbiente - MinEducación, 2002), es fundamental que las instituciones educativas tengan en cuenta el contexto socialcomunitario. Pues, dicho proceso debe contar con la participación de los diversos factores de la sociedad. 


\section{CIENCIAMATRIA}

Revista Interdisciplinaria de Humanidades, Educación, Ciencia y Tecnología

Año VII. Vol. VII. N¹. Edición Especial. 2021

Hecho el depósito de ley: pp201602FA4721

ISSN-L: 2542-3029; ISSN: 2610-802X

Universidad Nacional Experimental Francisco de Miranda (UNEFM). Santa Ana de Coro. Venezuela

Geidy Parejo-Blanco; Amarilis Martínez-Viloria; María-del-Carmen Jiménez-Barriosnuevo

Esto, a propósito de desarrollar una gestión ambiental adecuada y sostenible, que implique la realización de acciones conjuntas, basadas en políticas medioambientales de carácter rural y/o urbano. Es decir, las instituciones educativas deben posibilitar un trabajo educativo y cooperativo, entre la comunidad educativa, el gobierno e instituciones del sector y las organizaciones sociales, de manera que se evidencie un proceso participativo, responsable y ético de todos los factores sociales, capaz de generar conocimientos y capacidades para detectar, comprender y dar soluciones a las problemáticas ambientales (MinAmbiente - MinEducación, 2002).

Ahora bien, en el contexto de la EA, la formulación y desarrollo de proyectos ambientales como estrategia didáctica, debe asumirse desde un enfoque participativo y transformador, a partir de la comprensión de las necesidades y problemáticas que se dan en los contextos biofísicos y sociales. Ya que, solo así los diferentes actores podrán ir desarrollando una conciencia ciudadana que les permitirá hacer propuestas en conjunto, útiles, innovadoras y que impacten positiva e integralmente en el problema medioambiental (Tovar, 2017).

Entonces y como se ha dicho, en este contexto es fundamental la participación activa de diversos actores sociales en la construcción de proyectos educativos. En este sentido, Muñoz (2010) referenciado por Hernández \& Navarro (2018) y Ruiz \& Villa (2017) abordan la temática de redes educativas locales que se han de constituir partiendo de un enfoque de cultura colaborativa para la mejora y/o cambio escolar de manera colectiva. Pues, estas redes socialmente elaboran un sistema de relación y cooperación que hace que cada institución educativa trascienda de su recinto, para hacerse interinstitucional; a propósito de crear de manera participativa espacios y experiencias innovadoras de formación, gestión y acompañamiento de los proyectos compartidos que conlleven tanto al progreso de los centros educativos, como al desarrollo comunitario en conjunto.

Teniendo en cuenta lo anterior, es significativo resaltar algunos datos en torno a la situación de los PRAE, en diversas localidades colombianas. En tal sentido, la Secretaria 
de Educación de la Alcaldía Mayor de Bogotá (2008), con su estudio Diagnóstico del estado del PRAE realizado en 30 colegios públicos de la localidad de Engativa, enuncia que solo el $43.3 \%$, es decir trece (13) colegios, lo han desarrollado. No obstante, su construcción aparte de evidenciar falta de concordancia entre los objetivos y las estrategias planteadas, no responde totalmente a lo establecido en la normativa que lo reglamenta y fortalece, dado el desconocimiento que se tiene. Además, se observa desarticulado al PEI y con irrisoria incidencia sobre algunos componentes del currículo, limitando la transversalidad de una manera adecuada. En relación a la sostenibilidad de Ios PRAE, está incidiendo la carencia de recursos económicos y la falta de garantías en cuanto a los espacios, tiempos y talento humano, aunado a la necesidad que tienen de crear nuevas estrategias para fortalecer la participación y la vinculación de los diversos actores y al casi nulo proceso de evaluación y seguimiento, que permita lograr un mayor empoderamiento e impacto de los proyectos.

Alape \& Rivera (2016), considerando los resultados de su estudio diagnóstico sobre los proyectos ambientales escolares (PRAE), realizado con 48 colegios oficiales de básica y media de la ciudad de Bucaramanga, observan: primero, se excluyen muchos aspectos relacionados con la normativa reglamentaria; segundo, existe poca integración y participación de los distintos actores sociales en el proceso de diagnóstico y construcción del proyecto para atender las problemáticas medioambientales; tercero, las actividades responden básicamente a la celebración del calendario ambiental, convirtiéndolo en una acción activista de bajo impacto, pues difícilmente se generan espacios de discusión, de debate critico-reflexivo, aunado a que es poco lo que se hace en materia de evaluación y seguimiento; y, por último, es necesario el proceso de capacitación y acompañamiento permanente en todas las etapas de su desarrollo; pues, se evidencia que, aunque las instituciones educativas cuentan con un proyecto establecido, con su diseño y ejecución muestran estar fuera de contexto tanto a nivel normativo como pedagógico. 


\section{CIENCIAMATRIA}

Revista Interdisciplinaria de Humanidades, Educación, Ciencia y Tecnología

Año VII. Vol. VII. N¹. Edición Especial. 2021

Hecho el depósito de ley: pp201602FA4721

ISSN-L: 2542-3029; ISSN: 2610-802X

Universidad Nacional Experimental Francisco de Miranda (UNEFM). Santa Ana de Coro. Venezuela

Geidy Parejo-Blanco; Amarilis Martínez-Viloria; María-del-Carmen Jiménez-Barriosnuevo

Burgos (2017), a partir de su estudio realizado en 254 instituciones educativas de Boyacá sobre la situación de los PRAE, asegura que a pesar de que las instituciones educativas desde 1994 formalizaron la EA mediante la materialización del PRAE dentro de su proyecto educativo, la mayoría de estos se han formulado en los últimos 4 años, evidenciándose con ello que solo han sido una formalidad, pero sin un impacto fuerte en la cultura ambiental ciudadana. Esto, posiblemente se deba a situaciones como: la nula asignación presupuestaria, la formulación del proyecto con poca coherencia con la realidad situacional, la escasa participación de los actores sociales involucrados, la complejidad de hacerle seguimiento, ya que no tienen metas e indicador claros y la limitada articulación de los comités ambientales escolares, siendo necesario desarrollar metodologías que contribuyan a la correcta formulación e implementación del PRAE.

Huérfano (2018), con los resultados de su investigación sobre los Proyectos Ambientales Escolares (PRAE) cómo estrategia de implementación de la Educación Ambiental en la Educación básica y media, realizado en dos IED de la ciudad de Bogotá, enuncia que los docentes del área de ciencias naturales se constituyen en los actores principales y de mayor influencia, en el diseño y desarrollo de la diferentes acciones y actividades de los PRAE. Lo que pone en evidencia la limitada participación y articulación de los PRAE con otras áreas de aprendizaje.

Urdinola (2019) con su estudio analítico sobre la EA a partir de los PRAE de 10 instituciones educativas de Santiago de Cali, encontró que el profesorado no está lo suficiente preparado o familiarizado con el PRAE; por consiguiente, no tienen las herramientas necesarias para poner en práctica lo plasmado en ellos, aunado a que no existe involucramiento en el proceso, ni se evidencia un aporte innovador, por parte de los actores participantes, para diseñarlo, ejecutarlo y hacer del desarrollo sostenible del medioambiente un tema motivador e integrador. Asimismo, es palpable las deficiencias en los canales de información y comunicación, además, de la insuficiencia de tiempo y de recursos económicos para el buen desarrollo del PRAE. 
Con lo datado, se evidencia que las debilidades y dificultades observadas son coincidentes, en cuanto a la situación del PRAE de las diferentes instituciones educativas de las localidades abordadas por los investigadores, lo que conlleva a inferir que es eminentemente necesario reenfocar y acompañar los procesos de diseño y ejecución de dichos proyectos. Pues, estos se constituyen en estrategias de la educación para el cambio y la transformación de la realidad sociopolítica y cultural de la sociedad colombiana.

Ahora bien, en cuanto a la localidad de Santa Marta, capital del departamento de Magdalena y ámbito donde se desarrolla la investigación, es importante aportar información referente a su problemática ambiental como elemento de justificación para la presentación de innovadoras propuestas metodológicas para la construcción de proyectos ambientales en el contexto escolar. En tal sentido, Álvarez y Salazar (2014) argumentan que la ciudad de Santa Marta, presenta un déficit general en aspectos de saneamiento, infraestructura y seguridad, encontrándose significancia con la presencia de malos olores, aguas negras por comuna y liberación de aguas negras al mar con contaminantes y organismos patógeno, lo cual, es una práctica que afecta el medio ambiente. Asimismo, la actividad económica relacionada con el transporte de carbón, la existencia de solamente algunas calles pavimentadas, la escasez de aceras y de señalización adecuada, la ausencia de zonas verdes y espacios deportivos, suficientes para la cantidad de población que los requiere; representan algunas, de las múltiples situaciones que sustancia la problemática ambiental en la ciudad de Santa Marta.

Asimismo, Orjuela (2018), con su propuesta de estrategias ambientales y sanitarias para el manejo de residuos sólidos en unidades de paisaje tipo playa, en la localidad de Los Cocos de Santa Marta, muestra que la situación ambiental no ha variado mucho, pues, el diagnostico evidencia que la emisión de contaminantes a la atmósfera y la perdida de suelos, son factores que han generado un alto impacto al medio biótico. En cuanto a los residuos sólidos, se tiene que la localidad es altamente generadora de los mismos, al 


\section{CIENCIAMATRIA}

Revista Interdisciplinaria de Humanidades, Educación, Ciencia y Tecnología

Año VII. Vol. VII. N¹. Edición Especial. 2021

Hecho el depósito de ley: pp201602FA4721

ISSN-L: 2542-3029; ISSN: 2610-802X

Universidad Nacional Experimental Francisco de Miranda (UNEFM). Santa Ana de Coro. Venezuela

Geidy Parejo-Blanco; Amarilis Martínez-Viloria; María-del-Carmen Jiménez-Barriosnuevo

mismo tiempo, que presenta problemas en la gestión integral de residuos sólidos y la carencia de un sistema de aprovechamiento del material orgánico generado como residuo.

En este contexto, la Institución Educativa Distrital (IED) Once de Noviembre, ubicada en la comuna 6 de Santa Marta, la cual imparte formación integral a niños, jóvenes y adulto, en sus niveles de preescolar, básica, media técnica y nocturna, tiene un gran flujo de estudiantes de este sector de la ciudad. En tal sentido, se puede inferir poca conciencia ambiental entre los miembros de la comunidad, ya que, en el diario transcurrir de las actividades escolares se generan residuos sólidos, los cuales no son manejados de forma adecuada. Lo que constituye una de las principales problemáticas de la institución y las comunidades aledañas, debido a que esta población no está familiarizada con los procesos de cuidado ambiental y de tratamiento de los residuos sólidos, mediante técnicas de reciclaje o reusó.

Cabe decir, en esta IED, como centro piloto para el desarrollo del estudio, se han realizado esfuerzos por disminuir los efectos negativos ambientales y se ha desarrollado el PRAE tendiente a promover acciones positivas para el medio ambiente. Sin embargo, este no tiene un enfoque participativo que dé respuesta a las necesidades de la comunidad y, por lo tanto, su impacto no se generaliza a nivel sectorial.

Dada las condiciones que anteceden y considerando las observaciones hecha a dicha institución en el mes de marzo de 2021, con el propósito de indagar en torno a como se viene desarrollando el PRAE en el marco del programa de EA, se tiene que actualmente dicho proyecto, se enfoca principalmente en el plástico, por ser uno de los residuos solido con mayor impacto de daño en el medio ambiente, descalificando otras amenazas latentes en el sector. De igual forma el PRAE no está estructurado de acuerdo a los lineamientos nacionales, y las actividades planteadas no se ejecutan y en muchas ocasiones son improvisadas, es decir, no se encuentran relacionadas con los 
planteamientos del proyecto ambiental de la institución; lo que, de alguna manera, es un reflejo de las demás instituciones ubicadas en la comuna 6 de Santa Marta.

Aquí, es importante mencionar que la Secretaría de Educación de Santa Marta, ha venido realizando acciones por comunas con el fin de generar estrategias sectorizadas. Esto permite que las actividades se alineen con las problemáticas del sector, y, por tanto, tengan un mayor impacto en la comunidad, lo cual, se está realizando con diversos tipos de proyectos relacionados con el componente ambiental. Igualmente, para ella, es fundamental que estas propuestas se promuevan con estrategias didácticas basadas en las TIC. Pues, es necesario aprovechar las ventajas que otorga las tecnologías como recurso añadido, al generar pequeñas innovaciones educativas ad hoc a la metodología habitual en el ámbito formativo (Area, 2010). Sobre todo, en este momento crucial de estar viviendo una crisis sanitaria mundial por el Covid19, la cual ha conllevado a la suspensión de las actividades escolares a causa del aislamiento social declarado.

Todo lo planteado, propició el desarrollo de esta investigación a partir de cuestionar ¿Será necesario crear una red verde de trabajo participativo para la reconstrucción del PRAE?, de ser así, ¿Cómo crearla? De allí que, el propósito se enmarcó crear una red verde de trabajo participativo mediado por las TIC, que coadyuve a la reconstrucción de los PRAE, en las instituciones educativas de la comuna 6 de Santa Marta, Colombia; tomando como escenario piloto a la IED Once de Noviembre.

\section{METODOLOGÍA}

En la búsqueda del cambio y la transformación de los procesos sociales, políticos y culturales, es menester la participación activa de los diversos actores comunitarios. En tal sentido, la participación fortalece las posibilidades de transformación, al conllevar a un entendimiento más auténtico de la realidad que se vive y, con ello a un incremento de su capacidad de empoderamiento, autonomía y autodeterminación, tanto individual como colectivo (Abarca, 2016). 
Partiendo de esta premisa y considerando que es ineludible la tarea de reenfocar y acompañar los procesos de diseño y ejecución de los PRAE, el presente estudio se fundamentó desde un enfoque participativo como otra manera de producir conocimientos a la vez que se generan procesos de intervención social. Cabe destacar que todo proceso de intervención basado en este tipo de metodologías participativas, tiene como punto de partida la propia realidad y la experiencia de los individuos y grupos sociales que participan. Pues, solo así, se podrá forjar un proceso creativo de comprensión y reflexión de las creencias, actitudes, comportamientos y prácticas, que forman parte de su realidad cotidiana, hacerlas conscientes y retornar a dicha realidad con innovadoras formas de actuación práctica. De allí que Leis sostenga que en la metodología participativa:

El saber es un proceso vivo, dinámico, que se desarrolla en la interacción entre las personas, en su reflexión compartida sobre lo que hacen, lo que buscan, lo que aspirar y desean. Los conocimientos deben producirse en el propio proceso educativo. Esta producción no es individual, sino colectiva, a través del intercambio, el diálogo y la reflexión, relacionando la práctica y la teoría (Leis, 1989; p.36)

Ahora bien, es de saber que asumir los enfoques participativo en este contexto, implico hacer apropiación de Investigación Acción Transformadora (IAT) y su fundamento epistemológico de que la experiencia guiada por el proceso de acción-reflexión-acción (Ricoy, 2006), les permite a los participantes "aprender a aprender" Esto, en términos de Freire, es desarrollar la capacidad de descubrir su mundo desde una perspectiva críticareflexiva y, con ello, entender su papel en el proceso de transformación de su realidad social (Balcazar, 2003).

Como método de investigación y aprendizaje colectivo asumido, la IAT se orienta desde el principios esencial de resignificar a los sujetos en el escenario de la vida cotidiana, haciéndolos consciente de su mundo de sentidos y significados, de sus posibilidades como proyectos concretos de existencia y del compromiso para generar teorías contextualizadas, que lleven a repensar y reconstruir el hecho social, desde el diálogo 
que es capaz de instaurar el hombre como ser de acción en relación con otros, con su entorno, su cultura, su ambiente. Y, así, generar un cambio de creencia, de ser y de cosmovisión, para constituir un nuevo sistema social de relaciones, acciones y pensamiento en la sociedad (Zuluaga de Prato, 2012).

En cuanto al diseño del estudio, este se estructuro mediante las cuatro fases de la IAT acuñadas por Ander-Egg (2003) como: diagnóstico social participativo, planificación de propuesta para la resolución de problemas y toma de decisiones, puesta en marcha de las actividades planificadas y evaluación del proceso mediante la acción-reflexión-acción, acerca de lo que se está haciendo. Dichas fases se muestran a continuación:

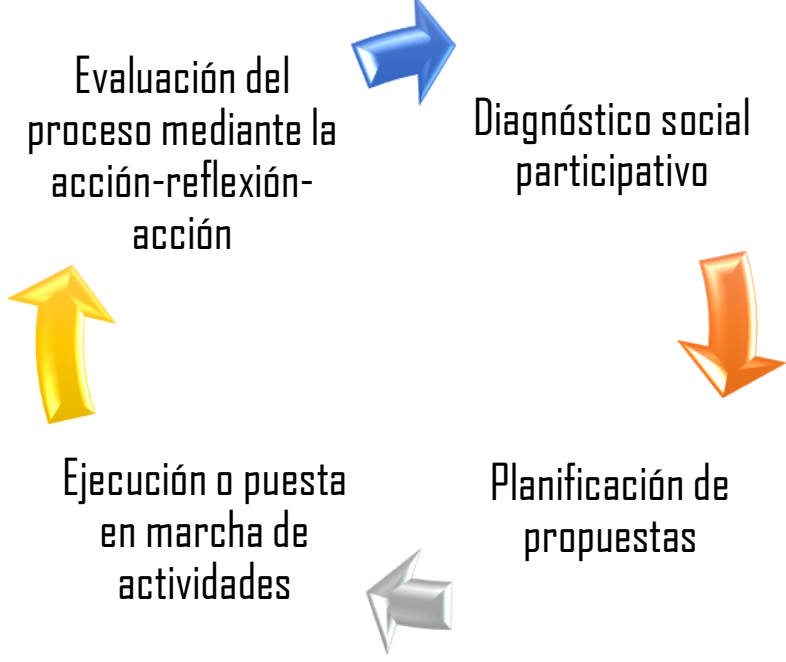

Figura 1. Fases de la IAP.

Fuente: Ander-Egg (2003). 


\section{CIENCIAMATRIA}

Revista Interdisciplinaria de Humanidades, Educación, Ciencia y Tecnología

Año VII. Vol. VII. N¹. Edición Especial. 2021

Hecho el depósito de ley: pp201602FA4721

ISSN-L: 2542-3029; ISSN: 2610-802X

Universidad Nacional Experimental Francisco de Miranda (UNEFM). Santa Ana de Coro. Venezuela

Geidy Parejo-Blanco; Amarilis Martínez-Viloria; María-del-Carmen Jiménez-Barriosnuevo

Respecto a los sujetos participantes co-implicados en el proceso que se desarrolla en esta etapa, pertenecen a la comunidad educativa de la IED Once de Noviembre. Es de saber que dicha institución, como escenario piloto, cuenta con 3256 estudiantes en los niveles de preescolar, básica, media académica y educación para adultos, en tres jornadas (mañana-tarde-nocturna), 7 sedes, 6 urbanas y 1 rural; 92 docentes, 1 rector, 6 coordinadores y 8 administrativos. Asimismo, la muestra de actores, como informantes clave y equipo de trabajo activo, fue seleccionada intencionalmente mediante los criterios cualitativos de mostrarse participativo, comprometido e interesado en el proceso (Goetz y Lecompte citados por Domingo, 2013). Por consiguiente, estuvo conformada por 30 estudiantes, 5 padres de familia, 5 docentes y 1 miembro de la Secretaria de Educación distrital.

Cabe resaltar que se asumió la selección y aplicación de técnicas e instrumentos tanto de corte cuantitativo como cualitativo. Es decir, desde un enfoque mixto, definido como aquel donde se aborda la realidad mediante un análisis cuantitativo y cualitativo de los datos recolectados, lo cual, permite un abordaje integral y holístico, ya que, se analiza el problema desde diversas perspectivas (Hernández, Fernández \& Batista, 2014). Las técnicas manejadas para recabar los datos consistieron en: la observación participante, la consulta a informantes clave, los grupos de discusión, el análisis documental y la encuesta. Utilizándose para ello: las notas de campo, el cuestionario, los registros descriptivos, el guion de preguntas abierta, todos ellos como instrumentos de investigación. En cuanto al análisis y procesamiento de los datos de tipo cuantitativo se hizo mediante la estadística descriptiva y la herramienta informática Excel, mientras que los de tipo cualitativo se dio a través del análisis de categorías y el software Atlas ti. 


\section{LOS RESULTADOS Y SU CONTRASTACIÓN}

Para dar inicio a la intervención en la IED Once de Noviembre como escenario piloto, previa constitución del equipo de trabajo participativo responsable del proceso, se solicitó ante la directiva un espacio para socializar los fundamentos del proyecto de intervención y los beneficios que pudiese lograr la institución frente a este. Lo planteado, permitió que se lograran desplegar todas las fases constitutivas de la IAP, en el marco de crear una red verde de trabajo participativo, que coadyuvara a la reconstrucción de los PRAE, desde un proceso sistemático de intervención (Folgueiras \& Sabariego, 2017). En vista de esto, se presentan los resultados obtenidos con el desarrollo de las distintas fases del proceso:

\section{Fase de Diagnostico Social Participativo}

Con la fase de Diagnostico Social Participativo inicia propiamente el proceso investigativo, pues, como expresa Silva (2005), aquí es donde se desvelan las necesidades sentidas por el grupo o comunidad abordada. A propósito de esto, se realizó una consulta a informantes clave, haciendo uso de un cuestionario de preguntas estructuradas, con el cual, se pudieron descubrir los hechos o situaciones que caracterizan al PRAE y la problemática ambiental percibida por los actores sociales de la IED Once de Noviembre, como participantes del proceso de intervención, tal como se muestra en la figura 2: 
$P 1$ - Nivel de conocimiento sobre el PRAE

$P 2$ - Existencia de afectación ambiental en la institución

P3 - Tipo de afectación ambiental en la institución

P4 - Transversalidad del PRAE

P5 - Áreas de aprendizaje que asumen el PRAE

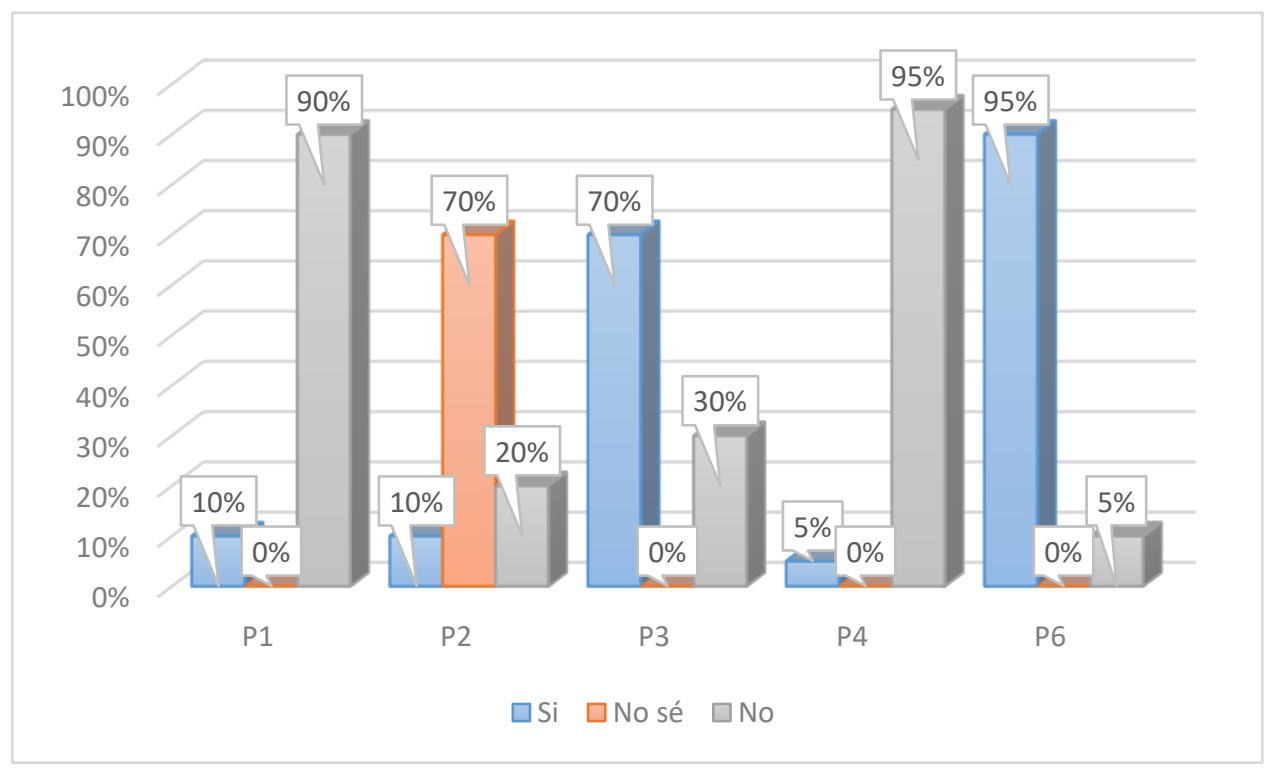

Figura 2. Conocimiento sobre el PRAE y la problemática ambiental percibida. Elaboración: Los Autores.

Con respecto a la pregunta 1, los resultados muestran que existe un alto nivel de desconocimiento sobre el PRAE como estrategia que entreteje la EA en el currículo, a través de propuestas pedagógicas, enmarcadas en atender los problemas medio ambientales a nivel local de manera participativa, ya que, el $90 \%$ los actores sociales consultados manifiestan desconocerlo. Este hallazgo se compagina con lo enunciado por Alape \& Rivera (2016), Burgos (2017) y Urdinola (2019), en el sentido de que los actores participantes no están lo suficiente preparado o familiarizado con el PRAE, pudiéndose inferir falta de conocimiento y pericia. Pues, los proyectos medioambientales se elaboran 


\section{CIENCIAMATRIA}

Revista Interdisciplinaria de Humanidades, Educación, Ciencia y Tecnología

Año VII. Vol. VII. N¹. Edición Especial. 2021

Hecho el depósito de ley: pp201602FA4721

ISSN-L: 2542-3029; ISSN: 2610-802X

Universidad Nacional Experimental Francisco de Miranda (UNEFM). Santa Ana de Coro. Venezuela

Geidy Parejo-Blanco; Amarilis Martínez-Viloria; María-del-Carmen Jiménez-Barriosnuevo

descontextualizado a lo normativo y pedagógico, dado que, carecen de coherencia con la realidad situacional, excluyen muchos aspectos en relación a la normativa que los reglamenta, hay desconocimiento en cuanto a los procesos de formulación, están desprovisto de seguimiento y ponen en evidencia que no existe un involucramiento real en el proceso, haciendo que la sostenibilidad ambiental sea un tema poco motivador a nivel educativo.

Atendiendo a los resultados que se presentan con las preguntas 2 y 3 , se tiene que el $70 \%$ de los actores sociales consideran que existen afectaciones ambientales en la institución, las cuales están relacionadas principalmente con el manejo de residuos sólidos, lo que evidencia que el PRAE, hasta ahora no se ha asumido como una oportunidad y una herramienta para su gestión integral, dado que se rescinde de otras amenazas latentes en el sector. Este descubrimiento se puede concatenar con lo expresado por Orjuela (2018), respecto a que, en las localidades costeras de Santa Marta como Los Cocos, se genera altamente residuos sólidos, al mismo tiempo que carecen de un buen sistema de gestión integral de aprovechamiento de material orgánico y residuos sólidos. Por lo cual es posible inferir que, en la localidad de Santa Marta, donde se encuentra esta institución, existe un patrón marcado del problema de los desechos sólidos, que requiere de un abordaje amplio a fin de generar un impacto institucional, pero, que trascienda a lo local.

Con los resultados de las preguntas 4 y 5 , se observa que el $95 \%$ de los actores sociales manifiestan que el PRAE no se ha asumido como una estrategia para la EA que se ha de transversalizar a todo el currículo, dado que las actividades de atención medioambiente no se están llevando a cabo de forma integradas a las distintas áreas de aprendizaje, ya que solo están involucradas con las áreas de ciencias naturales. Hallazgo que coincide con los manifestado por Huérfano (2018), en cuanto a que el PRAE está dirigido y liderado por los docentes de área de ciencias naturales, por lo cual, las actividades relacionadas en el PRAE se desarrollan prioritariamente por la influencia de esta área de 


\section{CIENCIAMATRIA}

Revista Interdisciplinaria de Humanidades, Educación, Ciencia y Tecnología

Año VII. Vol. VII. N¹. Edición Especial. 2021

Hecho el depósito de ley: pp201602FA4721

ISSN-L: 2542-3029; ISSN: 2610-802X

Universidad Nacional Experimental Francisco de Miranda (UNEFM). Santa Ana de Coro. Venezuela

Geidy Parejo-Blanco; Amarilis Martínez-Viloria; María-del-Carmen Jiménez-Barriosnuevo

aprendizaje. Asimismo, con lo expresado por la Secretaria de Educación de la Alcaldía Mayor de Bogotá (2008), en relación a que los PRAE, se observan desarticulados al PEI y con una irrisoria incidencia sobre algunos componentes del currículo, limitando la transversalidad de una manera adecuada. Para cerrar este apartado, cabe decir que los resultados que se obtuvieron en esta fase, muestran la necesidad que existe de reconstrucción del PRAE de manera más cónsona y articulados a lo normativo y lo pedagógico; asimismo, manifiestan la posibilidad de visualización de soluciones a las problemáticas detectadas y su estructuración mediante propuestas metodológicas innovadoras.

\section{Fase de planificación y Ejecución}

Teniendo los resultados del diagnóstico, es decir, cuando ya se sabe lo que acontece, es menester tomar decisiones en relación a lo que se va a hacer a fin de construir soluciones (Ander-Egg, 2003). En tal sentido, se presenta la propuesta de intervención planificada, de manera conjunta y colaborativa por los actores sociales participantes del proceso, mediante la técnica de grupos de discusión; al mismo tiempo, el desarrollo práctico de cada una de las estrategias planificadas, en virtud de lograr el objetivo de la intervención, pues como expresa el mismo Ander-Egg (2003), "Lo sustancial es pasar a la acción. Todo puede estar bien estudiado y planificado, pero ello no basta" (p.104).

Dicha propuesta; la cual, es un plan estructurado por objetivos (general y particulares), acciones estratégicas, participantes, tiempo, productos y/o resultados, esencialmente está relacionada con la creación de una red verde de trabajo participativo mediada por las TIC, que coadyuve a la reconstrucción de los PRAE en las instituciones educativas de la comuna 6 de Santa Marta, tomando como escenario piloto a la IED Once de Noviembre. Dicha propuesta presenta a continuación: 


\section{CIENCIAMATRIA}

Revista Interdisciplinaria de Humanidades, Educación, Ciencia y Tecnología

Año VII. Vol. VII. N¹. Edición Especial. 2021

Hecho el depósito de ley: pp201602FA4721

ISSN-L: 2542-3029; ISSN: 2610-802X

Universidad Nacional Experimental Francisco de Miranda (UNEFM). Santa Ana de Coro. Venezuela

Geidy Parejo-Blanco; Amarilis Martínez-Viloria; María-del-Carmen Jiménez-Barriosnuevo

\section{Tabla 1.}

Propuesta de Intervención.

\begin{tabular}{|c|c|c|c|c|c|c|}
\hline \multicolumn{7}{|c|}{$\begin{array}{l}\text { Objetivo General : Crear la "Red Verde" de Trabajo Participativo mediada por las TIC para la reconstrucción de los PRAES } \\
\text { en las instituciones educativas de la comuna } 6 \text { de Santa Marta, tomando como escenario piloto a la IED Once de } \\
\text { Noviembre }\end{array}$} \\
\hline $\begin{array}{c}\text { Acción } \\
\text { estratégica }\end{array}$ & $\begin{array}{c}\text { Descripción } \\
\text { general }\end{array}$ & Participantes & Tiempo & & $\begin{array}{l}\text { jetivo particular } \\
\text { que atiende }\end{array}$ & $\begin{array}{c}\text { Productos y/o } \\
\text { resultados }\end{array}$ \\
\hline $\begin{array}{l}\text { Diseño de ruta } \\
\text { y manual } \\
\text { digital para la } \\
\text { reconstrucción } \\
\text { de los PRAE }\end{array}$ & $\begin{array}{lr}\text { Tomando romo } \\
\text { referencia los } \\
\text { parámetros y } \\
\text { lineamientos } \\
\text { políticos - } \\
\text { gubernamentales } \\
\text { para la } \\
\text { construcción del } \\
\text { PRAE se diseña } \\
\text { la ruta que se } \\
\text { utilizará en las } \\
\text { actividades con } \\
\text { las instituciones. }\end{array}$ & $\begin{array}{l}\text { Equipo de } \\
\text { trabajo } \\
\text { participativo } \\
\text { Docentes } \\
\text { Estudiantes } \\
\text { Padres y } \\
\text { representantes } \\
\text { Personal } \\
\text { administrativo y } \\
\text { directivo }\end{array}$ & $\begin{array}{c}2 \\
\text { semanas }\end{array}$ & 1. & $\begin{array}{l}\text { Diseñar una } \\
\text { ruta para la } \\
\text { reconstrucción } \\
\text { del PRAE en las } \\
\text { IED de la } \\
\text { comuna } 6 \text { de } \\
\text { Santa Marta } \\
\text { teniendo en } \\
\text { cuenta los } \\
\text { parámetros } \\
\text { establecidos. }\end{array}$ & $\begin{array}{l}\text { Ruta y manual } \\
\text { digital para la } \\
\text { reconstrucción } \\
\text { del PRAE }\end{array}$ \\
\hline $\begin{array}{l}\text { Ejecución de } \\
\text { mesas de } \\
\text { trabajo para la } \\
\text { reconstrucción } \\
\text { del PRAE de la } \\
\text { IED Once de } \\
\text { Noviembre. }\end{array}$ & $\begin{array}{l}\text { Es una actividad } \\
\text { que buscan la } \\
\text { reconstrucción } \\
\text { participativa del } \\
\text { PRAE de la } \\
\text { institución, lo cual, } \\
\text { se hará utilizando } \\
\text { como insumo la } \\
\text { ruta diseñada. }\end{array}$ & $\begin{array}{l}\text { Equipo de } \\
\text { trabajo } \\
\text { participativo } \\
\text { Docentes } \\
\text { Estudiantes } \\
\text { Padres y } \\
\text { representantes } \\
\text { Personal } \\
\text { administrativo } \\
\text { y directivo }\end{array}$ & $\begin{array}{c}3 \\
\text { semanas }\end{array}$ & 2. & $\begin{array}{l}\text { Reconstruir el } \\
\text { PRAE de la IED } \\
\text { Once de } \\
\text { Noviembre de } \\
\text { forma } \\
\text { participativa } \\
\text { teniendo en } \\
\text { cuenta la ruta } \\
\text { diseñada. }\end{array}$ & $\begin{array}{l}\text { PRAE de la IED } \\
\text { Once } \\
\text { de Noviembre }\end{array}$ \\
\hline $\begin{array}{l}\text { Creación y } \\
\text { socialización de } \\
\text { la Red Verde y } \\
\text { la propuesta de } \\
\text { ruta como } \\
\text { estrategia para } \\
\text { la } \\
\text { reconstrucción } \\
\text { participativa de } \\
\text { los PRAE de las } \\
\text { IED de la }\end{array}$ & $\begin{array}{lr}\text { Posterior } & \text { al } \\
\text { establecimiento de } \\
\text { alianzas } \\
\text { educativas } \\
\text { interinstitucionales } \\
\text { con las IED de la } \\
\text { comuna } 6 \text { de } \\
\text { Santa Marta, se } \\
\text { desplegaran } \\
\text { grupos r de } \\
\text { discusión para } \\
\text { socializar la }\end{array}$ & $\begin{array}{l}\text { Equipo de } \\
\text { trabajo } \\
\text { participativo } \\
\text { Docentes } \\
\text { Estudiantes } \\
\text { Padres y } \\
\text { representantes } \\
\text { Personal } \\
\text { administrativo } \\
\text { y directivo }\end{array}$ & $\begin{array}{c}3 \\
\text { semanas }\end{array}$ & 3. & $\begin{array}{l}\text { Crear la "Red } \\
\text { Verde" de } \\
\text { trabajo } \\
\text { participativo } \\
\text { mediada por las } \\
\text { TIC, mediante } \\
\text { una alianza } \\
\text { educativa } \\
\text { interinstitucional } \\
\text { con las IED de } \\
\text { la comuna 6, } \\
\text { para la re- }\end{array}$ & $\begin{array}{l}\text { Acta de } \\
\text { conformación de } \\
\text { la "Red Verde" } \\
\text { Actas de } \\
\text { reuniones } \\
\text { mesas de } \\
\text { socialización. }\end{array}$ \\
\hline
\end{tabular}




\section{CIENCIAMATRIA}

Revista Interdisciplinaria de Humanidades, Educación, Ciencia y Tecnología

Año VII. Vol. VII. N¹. Edición Especial. 2021

Hecho el depósito de ley: pp201602FA4721

ISSN-L: 2542-3029; ISSN: 2610-802X

Universidad Nacional Experimental Francisco de Miranda (UNEFM). Santa Ana de Coro. Venezuela

Geidy Parejo-Blanco; Amarilis Martínez-Viloria; María-del-Carmen Jiménez-Barriosnuevo

\begin{tabular}{|c|c|c|c|c|}
\hline $\begin{array}{l}\text { comuna } 6 \text { de } \\
\text { Santa Marta. }\end{array}$ & $\begin{array}{l}\text { estrategia "Red } \\
\text { Verde" y la } \\
\text { propuesta de ruta } \\
\text { a seguir para la } \\
\text { reconstrucción de } \\
\text { los PRAE. }\end{array}$ & $\begin{array}{l}\text { IED de la } \\
\text { comuna } 6 \text { de } \\
\text { Santa Marta }\end{array}$ & 4. & $\begin{array}{l}\text { construcción de } \\
\text { los PRAE. } \\
\text { Socializar con } \\
\text { los miembros } \\
\text { de la "Red } \\
\text { Verde" la ruta } \\
\text { para la re- } \\
\text { construcción } \\
\text { participativa de } \\
\text { los PRAE de las } \\
\text { IED de la } \\
\text { comuna } 6 \text { de } \\
\text { Santa Marta. }\end{array}$ \\
\hline
\end{tabular}

Elaboración: Los autores.

Con el visto bueno de la rectoría, y atendiendo a la primera acción estratégica de la propuesta de intervención, se hizo una revisión documental de los aspectos conceptuales-teóricos y legales-normativos concerniente al PRAE. De igual manera, se dio paso al establecimiento de las mesas de trabajo participativo con el fin de diseñar una ruta para la reconstrucción del PRAE, en las IED de la comuna 6 de Santa Marta, teniendo en cuenta los parámetros establecidos. En este tenor, la figura $\mathrm{N}^{\circ} 3$ ilustra la estructuración de los pasos como ruta para la reconstrucción de los PRAE: 


\section{CIENCIAMATRIA}

Revista Interdisciplinaria de Humanidades, Educación, Ciencia y Tecnología

Año VII. Vol. VII. N¹. Edición Especial. 2021

Hecho el depósito de ley: pp201602FA4721

ISSN-L: 2542-3029; ISSN: 2610-802X

Universidad Nacional Experimental Francisco de Miranda (UNEFM). Santa Ana de Coro. Venezuela

Geidy Parejo-Blanco; Amarilis Martínez-Viloria; María-del-Carmen Jiménez-Barriosnuevo

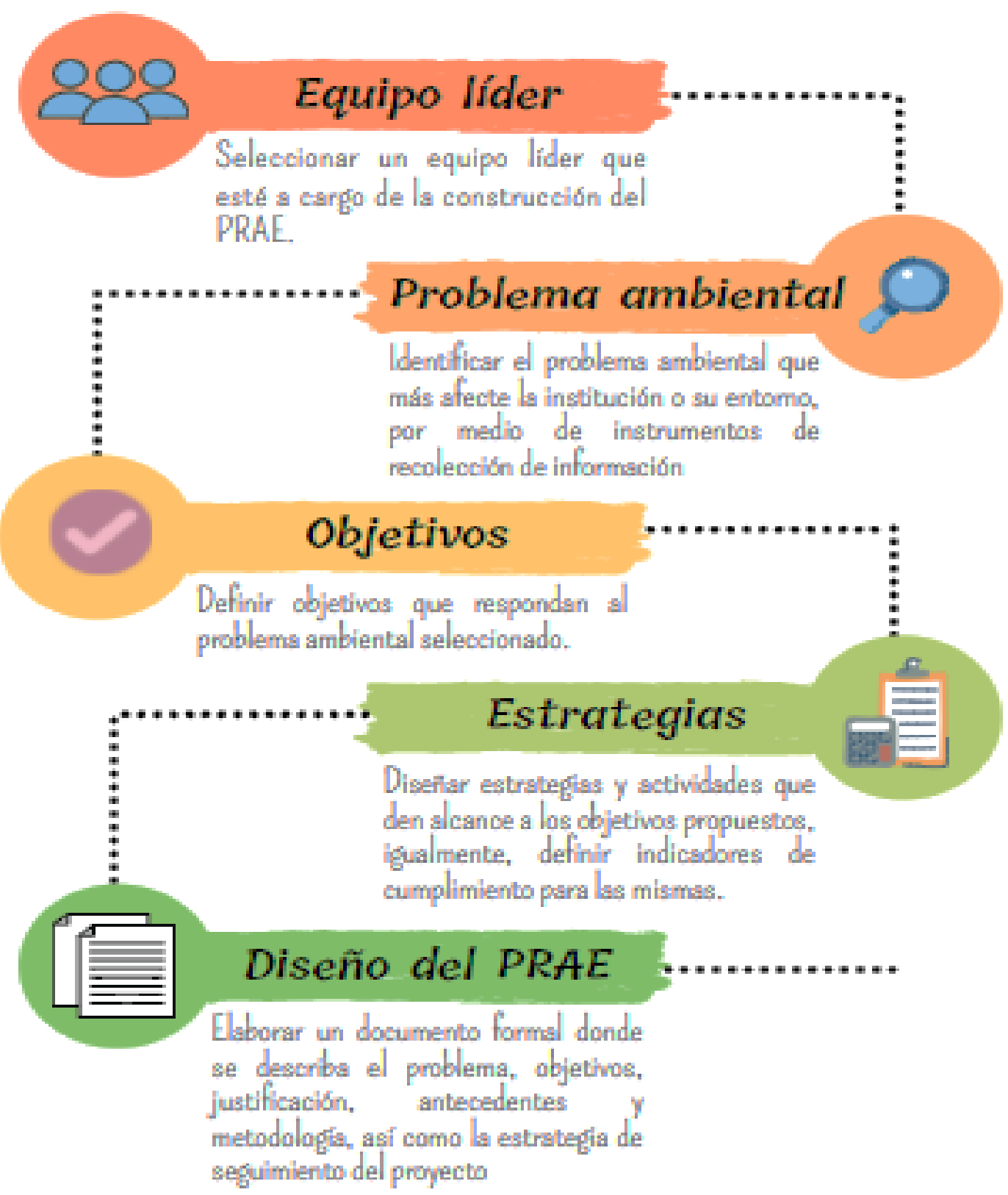

Figura 3. Ruta estructurada para la reconstrucción del PRAE. Elaboración: Los autores. 


\section{CIENCIAMATRIA}

Revista Interdisciplinaria de Humanidades, Educación, Ciencia y Tecnología

Año VII. Vol. VII. N¹. Edición Especial. 2021

Hecho el depósito de ley: pp201602FA4721

ISSN-L: 2542-3029; ISSN: 2610-802X

Universidad Nacional Experimental Francisco de Miranda (UNEFM). Santa Ana de Coro. Venezuela

Geidy Parejo-Blanco; Amarilis Martínez-Viloria; María-del-Carmen Jiménez-Barriosnuevo

En relación a la segunda acción estratégica, es de saber que se logro la reconstrucción del PRAE de la IED Once de Noviembre. En tal sentido, el proyecto, que se estructuro de manera articulada con el enfoque ecoturístico, se denomina: "Hacia una escuela ecoturística dinamizadora de procesos ambientales que contribuyan al desarrollo sostenible" Este proyecto se enmarca en incentivar las prácticas ambientales usando estrategias didácticas basadas en el ecoturismo, para dar adecuado manejo a los residuos sólidos y embellecer los paisajes. Desde esta perspectiva se organizaron actividades de socialización del PRAE, salidas de campo, campañas de reciclaje y muestras de decoración de paisajes y espacios con material reciclables. Además, se definió una estrategia de seguimiento que dé cuenta del cumplimiento de las actividades durante los plazos definidos.

En cuanto a la tercera y última acción estratégica, se alcanzó crear la "Red Verde" de trabajo participativo, mediante la realización de realizaron reuniones y mesas de socialización, para el establecimiento de alianzas educativas interinstitucionales con las IED de la comuna 6 de Santa Marta. Es de saber que las actividades estuvieron mediadas por las TIC, pues, pues, se buscó divulgar hacia las demás instituciones de la comuna, la experiencia participativa de reconstrucción del PRAE desarrollada en la IED Once de Noviembre. Asimismo, se compartió la ruta y el manual digital delineado para la reconstrucción de los PRAE, donde se exponen orientaciones a partir de la experiencia de la institución, aunado a que se creó un blog de información y socialización de las experiencias significativas desarrolladas por las IED de la comuna 6 integrantes de la red verde de trabajo participativo. 
Fase de Evaluación del proceso de intervención

La fase de evaluación fundamentalmente radica en valorar y reflexionar sistemáticamente los avances de las acciones y el logro de buenos resultados en torno a la propuesta de intervención que se ejecuta. Pues, solo en la medida que se aprende haciendo mediante el proceso de acción-reflexión-acción, se corrigen los errores y se mejoran las formas de actuación práctica (Ander-Egg, 2003).

Palpar las distintas situaciones vividas por los actores sociales durante el desarrollo de la intervención para la creación de la "Red Verde" de trabajo participativo mediada por las TIC, para la reconstrucción de los PRAES de las distintas IED de la comuna 6 de Santa Marta, permitió la emergencia de un conjunto de datos e informaciones que dan cuenta de la nueva realidad que acontece. Con estos datos, que representan los argumentos dados por los actores sociales y que emergieron con la aplicación de un cuestionario de preguntas abiertas aplicado de manera virtual, se hizo posible valorar el impacto de la estrategia. En tal sentido, se presentan los temas como categorías develadas, producto de su comprensión interpretativa realizada.

- Pertinencia pedagógica. La "Red Verde" de trabajo participativo mediada por las TIC, para la reconstrucción de los PRAES, se presenta como una estrategia pedagogía muy pertinente en el ámbito de la EA, en el sentido de que la creación de espacio de participación y la realización de las actividades enmarcadas en la crítica y reflexión, han fortalecido pedagógicamente el proceso de construcción del conocimiento en torno a los problemas medioambientales y como abordarlos de manera transversal mediante proyectos de investigación acción. Asimismo, tanto la ruta como el manual digital diseñado, se presentan de forma muy didáctica e ilustran de manera sencilla y entendible los pasos para reconstruir el PRAE. Esto permitió, que los distintos actores sociales hicieran una rápida apropiación de dichas herramientas. 
Lo planteado se relaciona con lo expresado por Huérfano (2018) y MinEducación (2005), en el sentido de que los PRAE, como estrategias de la EA entretejidas al currículo institucional, son generadoras de espacios de participación social que promueven habilidades de investigación, en torno a las problemáticas y las fortalezas medioambientales, desde una perspectiva citica reflexiva.

- Interrelación y comunicación. El desarrollo de la estrategia, ha sido altamente positiva, ya que, ha fomentado los procesos comunicacionales al coadyuvar a la creación de redes colaborativas, mediante el establecimiento de diálogos y de relaciones educativas interinstitucionales, que han permitido el despliegue de espacios de trabajo en conjunto, mediante el impulso de experiencias educativas y sociocomunitarias, para el tratamiento de los problemas ambientales $y$, la construcción y gestión de propuestas colectivas de solución.

Planteamiento que se correlaciona con las ideas de Hernández \& Navarro (2018) y Ruiz \& Villa (2017) a razón de que las redes educativas se constituyen en un eslabón fundamental en el desarrollo de procesos de planificación, gestión y formación conjunta que favorece a los centros educativos, al generarse interrelaciones que vigorizan la educación y dan cuenta de un alto nivel de sentido comunitario.

- Participación. El desarrollo de la experiencia de ha permitido el mejoramiento del interés de los distintos actores sociales. En tal sentido, la realización de acciones conjuntas con el trabajo en colectivo, ha fomentado tanto la generación de conocimientos y saberes en torno a los procesos y problemas del entorno, como la motivación y el deseo a participar activamente como miembro de la Red Verde y en las distintas actividades para la reconstrucción colaborativa de los PRAE y la creación de conciencia ambiental. Situación tal, se corresponde con lo referido por el MinAmbiente - MinEducación (2002), en torno a que generar conocimientos y capacidades para una adecuada y sostenible gestión de los problemas 
medioambientales, es esencial que las instituciones educativas consideren el contexto social-comunitario, a fin de que se desarrolle un trabajo educativo y cooperativo, que posibilite la participación y la realización de acciones colaborativa y en conjunto escuela-comunidad.

\section{REFLEXIONES FINALES}

Los PRAE, concebidos como estrategia pedagógica e instrumento que operacionaliza el entretejido de la dimensión ambiental y el currículo escolar de manera transversal, busca generar espacios de participación escuela-comunidad, para la investigación y la reflexión crítica de las dinámicas naturales y socioculturales, en pro de la transformación de la realidad medioambiental local que acontece. Pese a ello, con el proceso de diagnóstico social participativo desplegado en el marco de la intervención realizado a la IED Once de Noviembre, institución perteneciente a la comuna 6 de la localidad de Santa Marta; se descubrió que la afectación ambiental más evidente en la institución, atañe al manejo de residuos sólidos. Situación, de la cual se puede inferir, que es un patrón que se manifiesta en la localidad de Santa Marta.

En este contexto, los PRAE representan una vía para un abordaje amplio a fin de impactar tanto a nivel institucional, como local; sin embargo, se tiene que existe un alto nivel de desconocimiento sobre estos proyectos, por parte de los distintos actores educativos y sociocomunitario; aunado a que esta estrategia para el desarrollo de procesos de conocimiento de lo medioambiental, todavía no ha podido transversalizarse a las distintas áreas de aprendizaje en el currículo, teniendo a las ciencias naturales como área de mayor predominancia e influencia. Por ello, es importante que se generen procesos innovadores que conlleven a la reconstrucción de los PRAE, desde una nueva red de relaciones caracterizada por una cultura de trabajo participativo y colaborativo.

Considerando esto último, con el proceso de intervención se creo una red verde de trabajo participativo, mediado por las TIC, para la reconstrucción de los PRAE, en las 
instituciones educativas de la comuna 6 de Santa Marta, Colombia; tomando como escenario piloto a la IED Once de Noviembre. En tal sentido y con el proceso de evaluación realizado a las acciones de intervención se puede enunciar:

- La red verde de trabajo participativo mediada por las TIC y la ruta estructurada para la reconstrucción de los PRAE, se constituye en una estrategia de EA pedagógicamente pertinente para crear una cultura ambiental, que potencia la comprensión de los problemas desde la realidad contextual, los cuales, son producto de las dinámicas naturales y socioculturales, de manera colaborativa.

- La elaboración de herramientas digitales, como la ruta y el manual para la reconstrucción de los PRAE, representan ser instrumentos muy didácticos para hacer apropiación de conocimientos para una gestión pertinente de las problemáticas medioambientales.

- Reconstruir de forma participativa el PRAE de la institución, partiendo de la ruta diseñada, puso en evidencia que los actores sociales se hicieron conscientes de las problemáticas ambientales que los rodeaban. Esto promovió un sentido de reconocimiento de las falencias y desatinos que el proyecto tenia, principalmente en lo que respecta a la organización de las actividades y el seguimiento de las mismas. Asimismo, impulso que el proyecto asumiera la cualidad de ser transversal, al hacerse que las áreas de aprendizajes se integraran, mediante las distintas actividades didácticas focalizadas desde el manejo adecuado de residuos sólidos y el ecoturismo como alternativa sostenible, ambos aspectos importantes dentro del contexto ambiental.

- Para la reconstrucción de los modelos de gestión del PRAE, de manera participativa, colaborativa y en espacios de trabajo en conjunto, es fundamental el establecimiento de redes de comunicación y nuevas formas vinculación entre los actores de las distintas instituciones, a propósito de que se generen experiencias educativas y 
sociocomunitarias innovadoras, para el tratamiento de los problemas ambientales locales.

- La participación se constituye en un enfoque fundamental para la construcción del conocimiento social colectivo, a la vez que se generen procesos de intervención social. Los enfoques participativos buscan que los sujetos se redescubran en su ser, actuar y comportarse, desde la crítica y la reflexión, en el escenario de la vida cotidiana. Es decir, se transformen y, con ello, se reconstruyan socialmente los sistemas de relaciones, acciones y pensamiento que dominan los distintos ámbitos de la sociedad.

\section{REFERENCIAS}

Álvarez, L. \& Salazar, A. (2014). Características del ambiente urbano como determinante de salud en Santa Marta - Colombia. CES Salud Pública, (5),11-20. Recuperado de https://dialnet.unirioja.es/descarga/articulo/4804773.pdf

Alape, D. \& Rivera, M. (2016). Diagnóstico de los Proyectos Ambientales Escolares (PRAE) en Colegios Oficiales de Bucaramanga. (trabajo de maestría). Universidad Santo Tomas. Bucaramanga, Colombia. Recuperado de https://repository.usta.edu.co/handle/11634/1730

Ander-Egg, E. (2003). Repensando la investigación acción participativa. México. Editorial Lumen.

Area, M. (2010). El proceso de integración y uso pedagógico de las TIC en los centros educativos. Un studio de caso. Revista de Educación, 352, 77-97. Recuperado de http://www.revistaeducacion.educacion.es/re352/re352 04.pdf

Balcazar, F. (2003). Investigación acción participativa (IAP): Aspectos conceptuales y dificultades de implementación. Fundamentos en Humanidades, IV(7-8), 59-77. Recuperado de http://www.redalyc.org/articulo.oa?id=18400804

Burgos, A. (2017). Estado de los proyectos ambientales escolares en Boyacá. Revista Luna Azul, 44, 39-58. Recuperado de https://revistasojs.ucaldas.edu.co/index.php/lunazul/article/view/3825 
Bustamante, N., Cruz, M. \& Vergara, C. (2017). Proyectos ambientales escolares y cultura ambiental en la comunidad estudiantil de las instituciones educativas de Sincelejo, Colombia. Revista Logos Ciencia \& Tecnología, 9(1), pp. 215-229. Recuperado de https://www.redalyc.org/pdf/5177/517754057019.pdf

Chequea, N. \& Collazos, L. (2014). Formulación Participativa del Proyecto Ambiental Escolar (PRAE) para el Instituto Técnico Agropecuario y Forestal (ITAF) en Calima El Darién, Valle Del Cauca-Colombia. Recuperado de http://repositorioslatinoamericanos.uchile.cl/handle/2250/415100

Domingo, A. (2013). Practica Reflexiva para Docentes. Recuperado de https://n9.cl/bxjyb

Flórez, G. (2015). La educación ambiental y el desarrollo sostenible en el contexto colombiano. Revista Electrónica Educare, 19(3), 1-12. DOI http://dx.doi.org/10.15359/ree.19-3.5

Folgueiras, P. y Sabariego, M. (2017). Investigación-acción participativa. El diseño de un diagnóstico participativo. REIRE Revista d'Innovació i Recerca en Educació. Universidad de Barcelona, 11(1), 16-25. Recuperado de http://diposit.ub.edu/dspace/bitstream/2445/145200/1/677076.pdf

Hernández, E. \& Navarro, M. J. (2018). Redes educativas locales para la mejora escolar. Revista electrónica de investigación educativa, 20(3), 29-42. DOI https://doi.org/10.24320/redie.2018.20.3.1669

Hernández, R., Fernández, R., \& Baptista, P. (2014). Metodología de la investigación. Recuperado de https://n9.cl/65f

Huérfano. A. (2018). Proyectos Ambientales Escolares (PRAE) cómo estrategia de implementación de la educación ambiental en la educación básica y media en Colombia. (tesis de postgrado) Universidad Federal do Rio de Janeiro. Rio de Janeiro, Brasil. Recuperado de https://n9.cl/mkani5

Leis, R. (1989). El arco y la flecha. Recuperado de https://n9.cl/4kfsk

MinEducación. (agosto-septiembre, 2005). Educación ambiental: construir educación y país. Altablero. Recuperado de https://n9.cl/3samp 
MinAmbiente \& MinEducación (2002). Política Nacional de Educación Ambiental SINA. Recuperado de https://n9.cl/ca22

Orjuela, A. (2018). Desarrollo de estrategias ambientales y sanitarias para el manejo de residuos sólidos en unidades de paisaje tipo playa. Caso de estudio Los Cocos, Santa Marta. (trabajo de grado). Universidad de la Salle. Bogotá, Colombia. Recuperado de Obtenido de https://n9.cl/yhts6

Tovar, J. (2017). Pedagogía ambiental y didáctica ambiental: tendencias en la educación superior. Revista Brasileira de Educación, 22(69), 519-538. DOI https://doi.org/10.1590/S1413-24782017226926

Ricoy, C. (2006). Contribución sobre los paradigmas de investigación. Revista do Centro de Educação, 31(1), 11-22. Recuperado de https://www.redalyc.org/pdf/1171/1171117257002.pdf

Ruiz, G \& Villa, P. (2007). Propuesta para la conformación de red de proyectos ambientales (REDPRAE) en los corregimientos de Altagracia, Arabia, Estrella- La Palmilla y Morelia. (trabajo de grado). Universidad Tecnológica de Pereira. Pereira, Colombia. Recuperado de http://repositorio.utp.edu.co/dspace/handle/11059/857

Secretaria de Educación de la Alcaldía Mayor de Bogotá (2008). Diagnóstico del estado del Proyecto Ambiental Escolar (PRAE): Informe Cadel Engativa. Recuperado de https://n9.cl/7tt4v

Silva, E. (2005). Investigación Acción. Metodología Transformadora. Disponible en https://n9.cl/xfqg

Urdinola, S. (2019). Análisis de la Educación Ambiental a partir de los PRAE en la ciudad de Santiago de Cali. (tesis de pregrado). Universidad Autónoma de Occidente. Santiago de Cali, Colombia. Recuperado de https://n9.cl/loayst

Zuluaga de Prato, M. (2012). La investigación acción transformadora (I.A.T.): generadora de conocimiento desde lo colectivo popular. Investigaciones Interactivas COBAIND, $\quad I I(5) . \quad(s / p) \quad$ Recuperado de http://bdigital.ula.ve/storage/pdf/cobaind/v2n5/art5.pdf 


\section{CIENCIAMATRIA}

Revista Interdisciplinaria de Humanidades, Educación, Ciencia y Tecnología

Año VII. Vol. VII. N¹. Edición Especial. 2021

Hecho el depósito de ley: pp201602FA4721

ISSN-L: 2542-3029; ISSN: 2610-802X

Universidad Nacional Experimental Francisco de Miranda (UNEFM). Santa Ana de Coro. Venezuela

Geidy Parejo-Blanco; Amarilis Martínez-Viloria; María-del-Carmen Jiménez-Barriosnuevo

(C2021 por los autores. Este artículo es de acceso abierto y distribuido según los términos y condiciones de la licencia Creative Commons Atribución-NoComercial-Compartirlgual 4.0 Internacional (CC BY-NC-SA 4.0) (https://creativecommons.org/licenses/by-nc-sa/4.0/). 